\section{Cahiers de Narratologie}

Analyse et théorie narratives

$10.1 \mid 2001$

La voix narrative

\title{
Stratégies énonciatives et pratiques d'écriture journalistiques : le portrait dans Libération
}

\section{Véronique Fillol}

\section{(2) OpenEdition \\ 1 Journals}

\section{Electronic version}

URL: http://journals.openedition.org/narratologie/6974

DOI: 10.4000/narratologie.6974

ISSN: 1765-307X

\section{Publisher}

LIRCES

\section{Printed version}

Date of publication: 1 January 2001

Number of pages: 433-444

ISBN: 2914561032

ISSN: 0993-8516

\section{Electronic reference}

Véronique Fillol, "Stratégies énonciatives et pratiques d'écriture journalistiques : le portrait dans Libération", Cahiers de Narratologie [Online], 10.1 | 2001, Online since 19 November 2014, connection on 23 February 2021. URL: http://journals.openedition.org/narratologie/6974 ; DOI: https://doi.org/ 10.4000/narratologie.6974 


\title{
«STRATÉGIES ÉNONCIATIVES ET PRATIQUES D'ÉCRITURE JOURNALISTIQUES : LE PORTRAIT DANS LIBÉRATION »
}

\author{
Véronique FILLOL \\ Université d'Avignon
}

\begin{abstract}
«Il découle de tout ce que nous avons dit que le problème des formes de l'énonciation prise comme un tout acquiert une importance énorme. Nous avons déjà indiqué que ce qui manque à la linguistique contemporaine, c'est une approche de l'énonciation en soi." "

Mikhail Bakhtine (V. N. Volochinov), Le marxisme et la philosophie du langage : essai de la méthode sociologique en linguistique, 1929, (1977 pour la tr. fr.), p. 138.
\end{abstract}

"Qui raconte ? ", question qui concerne à des titres différents, le locuteur lambda, l'analyste du discours qu'il soit linguiste, sémioticien ou philosophe, ou encore le psychanalyste (sans oublier le lecteur).

La réflexion, en cours de construction, proposée ici s'inscrit dans le prolongement d'un travail de recherchel qui (re)situe la question de l'énonciation dans le cadre de l'analyse des discours, ou plus généralement de l'analyse des médias. Il s'agit de réexaminer les formes discursives et le rôle de l'énonciation dans la production des discours de la presse. Cette approche qui place l'énonciation comme stratégie et comme dispositif au cœur de la production de la signification

${ }^{1}$ Véronique FILLOL, Vers une sémiotique de l'énonciation : du lieu commun comme stratégie et des formes et/ou formations discursives comme lieux communs de l'énonciation énoncée (dans la presse féminine). Thèse de doctorat Nouveau Régime: Sc. Du langage, Université de Toulouse Le-Mirail, 1998. 
va à l'encontre d'une conception de l'énonciation « anthropoïde $»^{2}$.

\section{QUI est QUI ? : Qui est-il nécessairement un narrateur, une instance ou un sujet ?}

Si la réflexion de la voix narrative est une problématique qui ne cesse de "rebondir ", il en est de même pour ce qui est de l'énonciation. En Sciences du langage ${ }^{3}$, la question du sujet - longtemps évacuée - est revenue sur les devants de la scène au travers de la problématique de l'énonciation. L'énonciation étant - dans une définition originelle - à la fois l'acte (« la mise en fonctionnement de la langue par un acte individuel » dit Benveniste) et le résultat, le produit, on a pu distinguer tant en linguistique qu'en sémiotique, l'acte énonciatif et l'énonciation énoncée, étant entendu que si l'on veut rester dans le cadre d'une approche immanente des discours, la seule voie d'accès à l'énonciation est - de fait - l'énonciation énoncée.

Mais, dès lors que l'objectif relève de manière générale de l'analyse des discours sociaux, l'analyse ne peut s'en tenir à l'étude des acteurs textualisés (narrateurs et narrataires, actants narratifs), c'est-à-dire inscrits dans la matière textuelle de l'énoncé. On est ici contraint d'élargir le « cadre ", à savoir d'étudier "également les relations que ces figures textuelles entretiennent avec les figures présupposantes du niveau énonciatif (énonciateurs et énonciataires) ${ }^{4}$ et, au-delà, avec les

2 Terme emprunté à C. Metz in L'énonciation impersonnelle ou le site du film.

${ }^{3}$ Nous inscrivons explicitement notre réflexion dans le cadre des sciences du langage même si notre position se situe plutôt en «marges »: le terme renvoie ici à une différence de point de vue et évoque, dans le même temps, que les sciences du langage se sont décentrées du noyau historique classique pour s'inscrire dans de nouvelles relations interdisciplinaires.

4 C'est précisément le travail très intéressant proposé par Martin THUT dans son ouvrage : Le simulacre de l'énonciation: Stratégies persuasives dans Les Chants de Maldoror de Lautréamont, où M. Thut (dans la perspective des travaux de J. Geninasca) montre que la continuité du texte de Lautréamont est à situer au plan de 
acteurs sociaux "réels" inscrits dans le champ socioculturel général."5

Ce que l'on nomme "énonciation" n'est pas seulement l'objet de plusieurs conceptions différentes, mais contient en soi-même plusieurs idées distinctes. Deux de ces idées relevées dans le dictionnaire de Greimas et Courtés sont (i) l'énonciation comme une production, et (ii) l'énonciation comme passage, le passage d'une instance virtuelle (comme le code) à une instance réelle. "Mais il y en a une troisième, qui est même la première chez Benveniste et Jakobson et, dans le champ narratologique, chez Gérard Genette. Par énonciation, on désigne, aux deux « bouts » de l'énoncé, des personnes humaines, ou plus précisément des sujets."6

Si l'objet littéraire pose et impose une entrée dans l'analyse du jeu narratif et des stratégies énonciatives, il n'en va pas de même des discours de la presse. L'analyse des discours de la presse ré-interroge en ce sens la question des pratiques d'écriture mais aussi celle des points de vue : la façon de dire quelque chose peut être plus importante que le dit luimême. D'un point de vue de l'histoire de la théorie, l'étude des relations énonciatives et, notamment, la prise en compte de leur rôle dans une théorisation des médias est chose récente (Eliseo Veron : 1981, 1987). Cette réflexion a été prolongée dans le cadre d'une socio-sémiotique (Andréa Semprini : 1996). Nous résumerons le cadre de notre approche par la schématisation suivante :

l'énonciation, l'analyse se situant alors au niveau le plus immédiat qui est celui de l'énonciation énoncée.

5 Andréa SEMPRINI, Analyser la communication. L'Harmattan, 1996, p. 18.

${ }^{6}$ Christian METZ, L'énonciation impersonnelle ou le site du film. Paris : Méridiens Klincksiek, 1991, p. 11. 
Espace socio-culturel

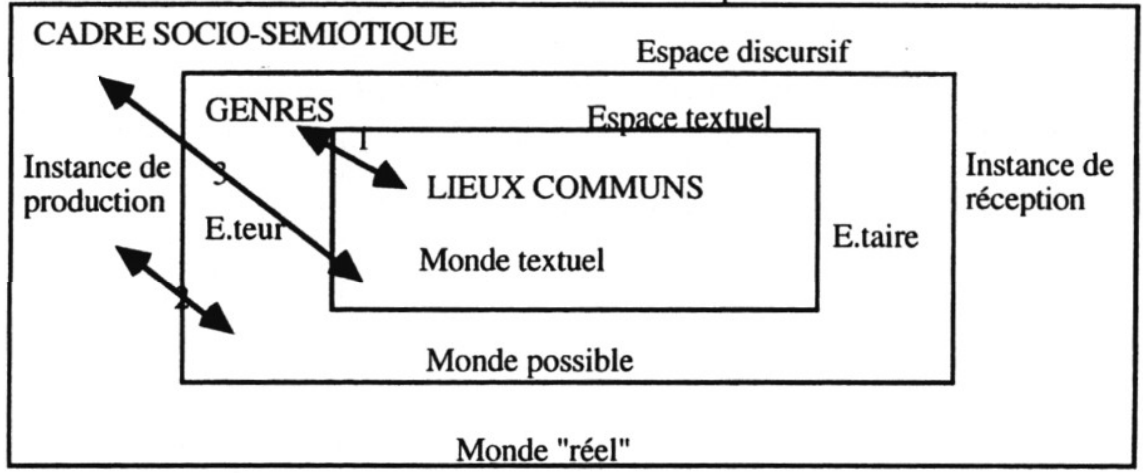

Cette réflexion tente de démontrer que l'énonciation (dans le sens de narration) dans la presse relève - sur un premier niveau d'analyse - d'une narrativisation du quotidien ou de l'actualité médiatique. Cette narration s'inscrit à son tour dans une position discursive au sein d'un genre (le genre est en soi une position discursive). Cette position discursive (analysable en termes de subjectivité ou de forme de vie) participe - en termes de stratégies discursives du positionnement identitaire du quotidien ou du magazine. Et cette construction d'une "d'identité discursive" fait sens et se justifie dans le champ de l'interdiscursivité sociale en termes de stratégie de communication médiatique.

Notre hypothèse est que l'analyse des discours de la presse ne peut se faire du côté du sujet, mais plutôt à partir du « lieu de parole $»^{7}$. L'examen de la source de parole doit être considéré dans le cadre établi dans lequel elle s'insère. Plus précisément, les phénomènes de polyphonie, de voix (qui intéressent le champ narratologique) et la dimension subjective (qui intéresse certaines approches du discours), doivent être analysés non du côté du sujet (quel que soit le statut que l'on donne à ce sujet) mais comme une position discursive à l'intérieur d'un genre. On ne peut parler du sujet sans

7 On rejoint ici d'une certaine manière la définition de la « voix » proposée par P. Hamon lors de la conférence plénière. 
considérer le lieu où il prend place. Ou, inversement, parler du sujet implique le cadre dans lequel il s'exprime, à savoir le genre. Parler d'une source de parole ou de source énonciative implique nécessairement - selon l'hypothèse avancée - d'analyser le cadre et ce cadre est le genre.

\section{Portrait d'un genre : le portrait dans Libération ${ }^{8}$}

Discours pluriel - selon la formulation de Landowski ${ }^{9}$ - le journal plus que tout autre discours social peut-être, se prête à une très grande diversité d'approches qui peuvent toucher aussi bien aux contenus idéologiques qu'aux structures narratives ou qu'aux stratégies de discours qui s'y manifestent. L'analyse que nous proposons ici a pour finalité de placer l'analyse de l'énonciation-narration au centre du dispositif énonciatif en termes (i) d'identité discursive, (ii) de stratégie de positionnement et (iii) de stratégie de communication médiatique.

La question que l'on se pose, c'est, bien sûr, la visée pragmatique en termes de contrat de lecture ${ }^{10} \mathrm{du}$ " portrait /profil » de Libération. Celui-ci occupe une place de choix au sein du dispositif d'ensemble du journal, en termes de visibilité. $\mathrm{Qu}^{\prime}$ en est-il de sa fonction dans la relation au quotidien avec le lecteur ? Participe-t-il du positionnement du journal, de son image de marque ? Notre hypothèse est que le portrait présenté en 4ème de couverture du journal configure la position du lecteur et produit dans le même temps un exercice (quotidien) de style : celui de "Libé », manière d'afficher et son image de marque et sa philosophie éditoriale.

${ }^{8}$ Nous avons sélectionné sur un ensemble de plus de 450 portraits depuis la création du portait en "4ème de couverture », 50 portraits de personnalités "médiatiques » différentes, c'est-à-dire personnalités politiques, médiatiques, comédiens, chanteurs sportifs, etc. pour n'en retenir que deux pour les besoins de l'analyse.

${ }^{9}$ La position d'analyse choisie par Landowski est encore autre et se situe dans une problématique plus globale, celle «qui vise le journal " tel qu'en lui-même », comme totalité de signification " (Une sémiotique du quotidien (Le Monde, Libération)", in La société réfléchie, p. 156).

10 Selon le sens que lui donne E. Veron dans ses travaux. 
Mais nous sommes ici au niveau des stratégies de communication médiatique, qu'en est-il des stratégies discursives ? La porte d'entrée de ce niveau énonciatif peut être le genre.

\subsection{Genre ou position discursive?}

Si le portrait ${ }^{11}$ n'est pas un genre «nouveau », mais bien au contraire un genre rédactionnel classique, sa généralisation et sa place au sein du dispositif médiatique du journal ou du magazine semblent récentes. Sa définition au sein de quelques manuels de journalisme ${ }^{12}$ le place tantôt dans la classe des genres rédactionnels de l'information, tantôt dans les genres du commentaire, ces deux classes de genre étant définis (par De Broucker) selon les critères (i) du sujet (point de vue sémantique), (ii) de l'intention (point de vue argumentatif) et (iii) de la position (niveau de l'énonciation). Ce qui nous permet d'avancer (à l'instar de Jean-Michel Adam avec qui nous sommes d'accord sur ce point) que les macro-«genres » distingués par De Broucker ${ }^{13}$ relèvent davantage d'une position énonciative par rapport à un contenu informationnel que de genres discursifs proprement dit.

Cette définition corrobore notre hypothèse, le genre - dans le cadre d'une théorie énonciative - est bien pour nous avant tout un cadre d'écriture (avec ses contraintes) et dans le même temps un positionnement énonciatif. Comment définir ce positionnement en termes de voix?

\subsection{Qui parle de qui ? Dialogue intersubjectif}

Dans la diversité des portraits de Libération, on retrouve une structure énonciative relativement stable (ou stabilisée) : l'énonciateur (qui est rarement le même journaliste ou écrivant) dresse un portrait d'une personnalité médiatique ou

11 «Article consacré à une personnalité, souvent illustré d'une photo » (Albert, 1989, p. 148).

12 On pourra se reporter à l'article introductif de Jean-Michel ADAM dans le numéro de Pratiques, n $^{\circ} 94$ (juin 1997) : "Unités rédactionnelles et genres discursifs : cadre général pour une approche de la presse écrite ", pp. 3-18.

13 José de BROUCKER, Pratique de l'information et écritures journalistiques, Paris, CFPJ, 1995. 
d'une personne qui devient «médiatique » du fait même de l'énonciation : c'est le cas par exemple du portrait de Yves Ferraud (37 ans, patron de Chez Francis, a vu sa paillote flamber et son chiffre d'affaires se consumer) dont la présentation diffère quelque peu du portrait quotidien (Une journée particulière : leur vie anonyme a croisé un événement important. Avant, après, récit). A noter que cette caractérisation explicite, voire métalinguistique du genre obéit au schéma narratif canonique décrit pas Courtés et Greimas (plus précisément à la transformation narrative : passage d'un état 1 à un état 2).

La finalité du portrait est, semble-t-il, d'inscrire une actualité médiatique (partagée par l'énonciateur et l'énonciataire présupposés puisque présente dans l'interdiscursivité médiatique) dans la narration de la vie du "personnage ». Cet événement est décrit dans le même temps comme un moment fort, une transformation narrative (en termes sémiotiques) dans son parcours ${ }^{14}$.

Cette configuration discursive, en quelque sorte réflexive (l'événement médiatique fait partie de la vie du personnage, et peut de ce fait être médiatisé, et réflexivement celui-ci devient un élément de la narration proprement dite), relève d'une stratégie énonciative intéressante.

A noter qu'on peut aussi rendre compte au niveau de la narration, de jeux subtils et de rapports complexes entre le personnage privé et le personnage médiatique, particulièrement lorsque celui-ci est comédien ou écrivain. On rentre alors dans un vertige de rôles actanciels, de jeux de rôles et de points de vues. Le narrateur ne se contente pas de relater la vie du personnage, il place le «je » dans un système de citations explicites où sont rapportées ses propres paroles, mais aussi celles de l'entourage privé ou professionnel du personnage. Mais ce portrait - entre subjectivité et effet d'objectivité - n'est pas seulement une narration. Celui-ci doit au final pouvoir représenter une figure ou un personnage

14 Exemple: Yves SIMON, 55 ans, écrivain, chanteur, humaniste, rêve des jeunes filles. Il sort demain son nouvel album, « intempestives ». 
suffisamment construit et cohérent pour avoir sa place au sein de l'espace médiatique. On trouve alors comme deuxième spécificité du portrait de Libération un nombre important de références externes à son discours qui permet de le situer luimême dans un ensemble de représentations partagées.

Quelle est, plus précisément, la construction discursive (ou stratégie énonciative) du portrait de Libération? Pour l'ensemble des portraits étudiés, on retrouve ainsi un dialogisme $^{15}$ au sens fort du terme, à savoir les deux stades distingués par Bakhtine d'empathie et d'exotopie (traduit par Todorov), l'empathie étant le stade de l'identification (le romancier se met à la place de son personnage), et l'exotopie, celui du mouvement inverse, par lequel il réintègre sa position.

L'énonciateur du portrait dans Libération obéit - semble-til - à ce double mouvement. La vie du « personnage » médiatique est rapportée selon des termes qui pourraient être ceux du personnage lui-même, selon ces mots à luii ${ }^{16}$. Ce discours citant, - pour utiliser une formulation neutre - ce discours racontant englobe le discours cité (nombreuses citations, c'est-à-dire " discours direct »), dont tantôt il s'imprègne tantôt se distingue. Cette écriture, cette énonciation dialogique construit une certaine distance.

Cette stratégie de la distance construit à la fois un effet d'objectivisation et un effet de complétude. L'énonciateur (comme l'auteur pour Bakhtine) ne pourra accomplir,

15 «La vie est dialogique, de par sa nature. Vivre signifie participer à un dialogue, interroger, écouter, répondre, être en accord, etc. " Cité par Todorov in "Bakhtine et l'altérité », Poétique, n 40 , nov. 1979 : p. 504.

16 D'où l'importance de commentaires métalinguistiques sur la parole du «personnage »: "rit-elle dans ce français légendairement parfait jusqu'à la maîtrise de l'argot " (Jodie Foster, 17/09/97) ; « Nathalie Baye égrène ses phrases sans s'attarder » $(04 / 09 / 98)$ « (...) Christine Angot se révèle bavarde. Non qu'elle se livre au jeu codifié, du portrait (et votre enfance, et vos souvenirs, et vos anecdotes), ce serait trop inconvenant (...) Christine Angot parle, et ça commence souvent ainsi : "Voilà, je sais, je peux dire... »" (29/06/99) une description de son rapport à la langue, de son rapport au monde par le discours ou encore de son rapport à l'autre dans le dialogue. 
achever, clore son personnage, que s'il lui est extérieur ; il est cet autrui porteur des éléments transgrédients dont le personnage a besoin pour être complet ${ }^{17}$. Pour que le portrait ait une existence discursive, il faut que le personnage soit complet, soit un tout accompli, et c'est l'auteur (énonciateur) qui lui fournira les éléments manquants indispensables.

Dans la deuxième partie du mouvement, celui de l'exotopie, il est un deuxième élément qui participe de la distance de cet effet d'objectivisation, ce sont la présentation systématique de dates, d'événements dans une chronologie collective. Et l'on retrouve alors au centre la temporalité narrative et la temporalité objective, le temps de l'énonciation qui est celui du quotidien.

Cette structure narrative est selon nous au cœur du dispositif énonciatif du "portrait » de Libération. Ce double mouvement brièvement décrit ici permet en effet d'intégrer une parole « autre » à la matière textuelle. Le rôle du narrateurénonciateur est profondément actif, mais son action a un caractère dialogique particulier. Il anticipe, il résume, il reprend, il interrompt souvent la voix de l'autre mais il ne la couvre jamais, il ne la termine jamais à partir de soi, c'est-àdire d'une conscience étrangère (la sienne).

Le positionnement de Libération relèverait ainsi d'une « sémiotique du mélange » (vs « sémiotique du tri »). La position est celle d'un "entre-deux », sorte de compromis entre le respect des contrats génériques et la mise en scène de registres verbaux et de formes discursives marginaux. Cette « rhétorique de la bonne distance » est intéressante du fait qu'elle permet, à la fois un effet d'objectivisation et de subjectivisation, et un lien socio-culturel (la doxa) et qu'elle déploie enfin, une configuration discursive complexe: humour, cynisme, dérision, etc.

\section{Conclusion : relations énonciatives, genres et stratégies de communication sociale.}

Pour conclure, une telle perspective permet de rendre compte du «je» en dehors d'une conception anthropoïde de

${ }^{17}$ Op. cit., p. 503. 
l'énonciation. Le «qui »-dans une définition communicationnelle de l'énonciation pourrait retrouver son nom d'auteur, mais alors au sens de qui «fait autorité », le « ici » sur cette même dimension de la communication sociale est l'espace même de réception des discours et le "maintenant " est bien sûr la temporalité contemporaine (proche de la notion de mode).

Au niveau des stratégies énonciatives, le « je » est bien plus une position discursive, ou si l'on conserve la dénomination de « je », il s'agit d'un sujet à l'intérieur d'un cadre, celui du " genre », cette position définissant à la fois le je et le ici dans la quotidienneté du journal. Et, enfin, c'est au niveau textuel que la variation du je est la plus importante.

A titre d'ouverture, il nous semble tout aussi intéressant (parallèlement à l'étude des genres) de développer l'étude de l'hétérogénéité énonciative dans le cadre d'une approche des médias. S'il est commun de mesurer et d'analyser l'altérité déclarée d'un texte, d'un discours journalistique, médiatique, qu'en est-il de l'hétérogénéité des discours qui coexistent à l'intérieur d'un journal ou d'un magazine ? Et par là même, comment la multiplicité de ces "genres discursifs" construitelle une pratique (ou un sens) de lecture ? Ou en d'autres termes - comment à ce niveau de pertinence - rendre compte de la cohérence d'un magazine en tant qu'ensemble hétérogène de discours. Quel est le "liant" ? Quel est le processus isotopant au niveau énonciatif ? Partir de la problématique de l'énonciation, et plus précisément s'interroger sur l'espace de réception de tout discours est une perspective intéressante : "les médias, situent désormais virtuellement tout discours dans une place publique - ou plus précisément dans un espace de réception - de plus en plus vaste ${ }^{18}$.

18 Andréa SEMPRINI, Le Multiculturalisme, P.U.F., coll. "Que SaisJe ?", 1997, p. 48. 


\section{BIBLIOGRAPHIE}

BAKHTINE (Mikhail) : 1963. Problemy poetiki Dostoïevkovo. Ecrivains soviétiques: Moscou. (Tr. fr. La poétique de Dostoievski. Paris : Seuil. 1970.

1977. Le marxisme et la philosophie du langage. Paris : Minuit.

DEPRETTO (Catherine) : Eds. 1997. L'héritage de Bakhtine. Presses Universitaires de Bordeaux.

FILlOL (Véronique): 1999. "Énonciation et usage : l'impersonnel de l'énonciation ou le jeu discursif dans les brèves de la presse féminine ». in Littératures, $\mathrm{n}^{\circ} 20$, "Sociocritique et analyse du discours », (Montréal), 1999, pp. 39-57

1998. Vers une sémiotique de l'énonciation: du lieu commun comme stratégie et des formes et/ ou formations discursives comme lieux communs de l'énonciation énoncée (dans la presse féminine). Thèse de doctorat Nouveau Régime: Sc. du langage, Université de Toulouse II, 1998.

FISCHER (Sophie) et VERON (Eliséo) : 1986. "Théories de l'énonciation et discours sociaux ». IN Etudes des lettres, Genève, Oct., 71-92.

LANDOWSKI (Éric) : 1989. La société réfléchie. Paris : Seuil. 1993. "On ne badine pas avec l'humour: la presse politique et ses petits dessins ». In Humoresques, 4.

1997. Présences de l'autre. Paris : Presses Universitaires de France.

Pratiques, ${ }^{\circ}$ 94, «Genres de la presse écrite ». Metz, 1997.

SEMPRINI (Andréa) : 1996. Analyser la communication. Paris : L'Harmattan. 
TODOROV (Ttzvetan) : 1970. «Problèmes de l'énonciation ». IN Langages, 17, Mars, 3-11.

1981. Mikaïl Bakhtine : le principe dialogique. Paris : Seuil.

VERON (Eliséo) : 1983b. «Quand lire, c'est faire : l'énonciation dans le discours de la presse écrite». IN "Sémiotique II", IREP (Institut de Recherches et d'Études Publicitaires), 33-56.

1985. "L'analyse du "contrat de lecture" : une nouvelle méthode pour les études de positionnement des supports presse". IN "Les médias: expériences, recherches actuelles, applications", IREP, 203-230.

1995. "Médiatisations du pouvoir. Stratégies - acteurs et constructions des collectifs ». In Hermès, 17-18, ED. CNRS. 